\title{
Using Eye Tracking and Electroencephalograph to Understand the Efficacy of Digital and Static Outdoor Advertisements
}

\author{
Reas, Brandon; Dishman, Paul; McCarter, Aaron; Jolley, Alan Dale \\ Vivint Neuromarketing SMARTLab, Utah Valley University, USA.
}

\begin{abstract}
This study discusses the contributions of advanced eye tracking research combined with electroencephalography (EEG) as a method of understanding the cognitive processing of digital vs. static outdoor advertisements. Subjects were exposed to a variety of billboard advertisements on a section of Interstate freeway in a suburban area in the western United States. Results showed that visual fixation time was higher for digital advertisements compared to static advertisements. In particular, the eye-tracking data revealed which advertisements received the most attention. This was mainly dependent upon location (i.e. distance from driver, distance from adjacent traffic signs, etc.).
\end{abstract}

As eye-tracking systems have become more sophisticated and affordable, there has been an increasing interest in the use of eye tracking within the traffic safety and outdoor advertising domain (Perez \& Bertola, 2010). Eye tracking studies that have focused on web-based and driving stimuli have gathered eye-movement data while participants were engaged in lowattention settings (Lee and Ahn, 2012). The findings of these studies have indicated that digital and animated advertisements, in low attention settings, reduce the likelihood of mental recall and result in overall decreased cognitive engagement.

Twenty-five subjects between the ages of 18 and 45 participated in the study. A 16 mile (25.75 kilometer) freeway drive was videotaped and then projected onto a four-by-six-foot screen. Subjects then viewed the projected video from inside a stationary car to simulate a driving environment. Using Tobii2 Glasses eye tracking system, subjects' eye-movements and gaze patterns were recorded during the simulation. EEG data was also collected to measure the subject's emotional response, and to gain additional insight into how they felt about the advertisements. In addition, participants were asked immediately after their drive to list any advertisements they recalled. 
These findings provide indications for best practices of effective outdoor advertising using gaze pattern analysis. These include positioning, layout, color schemes, etc. A potential implication for digital advertisements could be identifying the optimal length of time to display digital signage. Additionally, the results may suggest improvements in specific industry ads in order to maximize cognitive influence on consumer action (e.g. best times to display food and beverage advertisements).

From a traffic safety consideration, these results will provide a psychological understanding of whether or not outdoor advertisements present safety implications to drivers. Overall, findings provide a better understanding of digital and static outdoor advertising as it relates to safety and consumer behavior. The results of this study may have significant implications in both the private and public sectors.

Keywords: Outdoor Advertising; Eye tracking; Electroencephography; Billboards. 